Editorial

\title{
Computational Finance
}

\author{
Lars Stentoft ${ }^{1,2}$ iD \\ 1 Department of Economics, University of Western Ontario, London, ON N6A 5C2, Canada; \\ lars.stentoft@uwo.ca \\ 2 Department of Statistical and Actuarial Sciences, University of Western Ontario, London, \\ ON N6A 5B7, Canada
}

Received: 24 June 2020; Accepted: 1 July 2020; Published: 4 July 2020

\begin{abstract}
The field of computational finance is evolving ever faster. This book collects a number of novel contributions on the use of computational methods and techniques for modelling financial asset prices, returns, and volatility, and on the use of numerical methods for pricing, hedging, and risk management of financial instruments.
\end{abstract}

Keywords: asset pricing; calibration; derivatives; hedging; multivariate models; risk management; simulation; volatility

In a Special Issue of the Journal of Risk and Financial Management, there was a call for contributions within the broad topic of Computational Finance. The topic includes novel research on the use of computational methods and techniques for modelling financial asset prices, returns, and volatility, and in the pricing, hedging, and risk management of financial instruments. Theoretical and empirical articles on the application of novel computational techniques in estimation, simulation, optimization, and calibration with applications to asset pricing, derivative valuation, hedging, and risk management were welcomed and contributions focusing on multivariate or high-dimensional applications in today's complex world, novel measures of financial risk, and other types of risks implied from derivative markets, and on the use of high-frequency data of all sorts, were especially encouraged.

This book contains 10 contributions appearing in this special issue. A first group of papers contain new multivariate models, see Cheng et al. (2019), and novel techniques for pricing derivatives in such flexible models, see Reesor and Marshall (2020), and examines how flexible valuation methods based on simulation and regression techniques, see, e.g., Longstaff and Schwartz (2001), can be improved upon in general, see Létourneau and Stentoft (2019), and specifically when it comes to pricing realistic and relevant long maturity real options, see Stentoft (2019). A second group of papers examines how pricing and hedging techniques can be used to asses the challenges faced by insurance companies by changing regulatory requirements, see Van Dijk et al. (2018), by pension plan participants due to the increased use of defined contribution plans, see Forsyth and Vetzal (2019), and by market participants in general as a result of the increased sovereign linkages, see Dunne (2019). Finally, the last group of papers considers in one way or another issues related to high-frequency trading in general, see Mukerji et al. (2019), to the use of statistical arbitrage strategies in particular, see Stübinger and Schneider (2019), and to the use of high-frequency data to asses risk and volatility in financial markets, see Petrov et al. (2019).

Cheng et al. (2019) introduce a novel multivariate mean-reverting stochastic volatility model obtained by combining recently developed univariate volatility models with factor models using a principal component and factor covariance decomposition. The model is referred to as a multivariate Generalized Mean-Reverting 4/2 Factor Model, a name that comes from the superposition of a CIR term and a 3/2-model component. Compared to other existing multivariate stochastic volatility 
models the setting used reduces the dimension of the parametric space making popular estimation methods feasible. The authors find conditions for well-defined changes of measure and develop closed form expressions for two key characteristic functions, which allow for fast and efficient calculation of derivatives prices and risk measures and exposures, and they demonstrate numerically the significant impact of their model, both with respect to changes on the implied volatility surface and on two standard risk measures.

Reesor and Marshall (2020) present a new numerical technique for pricing multiple exercise options by simulation referred to as the Forest of Stochastic Trees (FOST) method. The proposed method uses stochastic trees in place of binomial trees in the Forest of Trees algorithm originally proposed to value swing options and extends this algorithm to allow for a multi-dimensional underlying process. The method can also be viewed as extending the stochastic tree method for valuing (single exercise) American-style options to multiple exercise options. The authors demonstrate that the proposed valuation method results in low and high biased estimators for the true option value and that these estimators are consistent for the true option value. The proposed method is of particular use in cases where there is potentially a large number of underlying assets and/or where the underlying price process depends on multiple risk factors.

Létourneau and Stentoft (2019) also consider option pricing but uses instead simulation and regression based techniques, see, e.g., Longstaff and Schwartz (2001). The authors propose an algorithm that improves on the approximation of the optimal early exercise boundary for American options. The method works by exploiting and leveraging the information in multiple cross-sectional regressions by averaging the individually obtained estimates at each early exercise step in the backwards induction algorithm. With this method, less errors are accumulated, and as a result of this, the price estimate is essentially unbiased even for long maturity options. Moreover, because the method naturally disassociates the estimation of the optimal early exercise boundary from the pricing of the option, significant efficiency gains can be obtained by using less simulated paths and repetitions to estimate the optimal early exercise boundary than with the regular method.

Stentoft (2019) also considers the LSM method of Longstaff and Schwartz (2001) and uses put-call symmetry to estimated call prices by pricing these as symmetric put options instead. His results show that, for a large sample of options with characteristics of relevance in real-life applications, the symmetric method performs much better on average, is the best method for most of the options, never performs poorly and, as a result, is extremely efficient compared to the optimal, but unfeasible method that picks the method with the smallest Root Mean Squared Error. The relative improvements of using symmetric pricing increases with option maturity and with asset volatility, and using the method to price, for example, real options, many of which are call options with long maturities on volatile assets, for example energy, could improve the estimates significantly.

Using option pricing theory, Van Dijk et al. (2018) consider the challenges faced by insurance companies when it comes to valuing issued guarantees at a one year horizon as is required by recent changes in regulation. These guarantees have option like features and the standard approach is to assume that the parameters of these option pricing models are constant, i.e., the calibrated parameters from time $t=0$ are also used to value the guarantees at $t=1$. However, it is well-known that the parameters are not constant and may depend on the state of the market. The authors propose an improved regression model that, given a set of market variables such as the VIX index and risk-free interest rates, can be used to estimate accurately the calibrated parameters at future dates. They show that, depending on the initial state of the market, the impact on the Solvency Capital Requirement may vary between $-46 \%$ and $52 \%$.

The increased use of Defined Contribution (DC) plans implies that more pension plan participants will bear the risk that final realized portfolio values may be insufficient to fund desired retirement cash flows. Forsyth and Vetzal (2019) compare the outcomes of various asset allocation strategies for a typical DC plan investor in a synthetic market and also using bootstrap resampling of historical data. They propose a strategy based on optimal dynamic (multi-period) time consistent quadratic 
shortfall and demonstrate that the probability that portfolio values at retirement will be insufficient to provide adequate retirement incomes is relatively high, unless DC investors adopt optimal allocation strategies and raise typical contribution rates. The results suggest that there is a looming crisis in DC plans, which requires educating plan holders in terms of realistic expectations, required contributions, and optimal asset allocation strategies.

The above contributions rely on having access to safe or risk free assets in combination with risky assets to generate sufficient cash flows. Dunne (2019) considers the benefits and disadvantages of introducing a European Sovereign Bond-Backed Securitisation (SBBS) to address the need for a common safe asset that would break destabilising bank-sovereign linkages. The author uses a simulation approach to assess the effectiveness of hedges incurred while making markets in individual euro area sovereign bonds by taking offsetting positions in one or more of the SBBS tranches. He finds that optimal hedging with SBBS reduces risk exposures substantially in normal market conditions. In volatile conditions, hedging is not very effective but leaves dealers exposed to mostly idiosyncratic risks which disappears if dealers are diversified in providing liquidity across country-specific secondary markets and SBBS tranches.

Mukerji et al. (2019) also use simulation methods, though at a high frequency, to study the impact of algorithmic trading (AT) in a market in which human and algorithmic counterparts trade based on technical and fundamental analysis and statistical arbitrage strategies. Considering variations in the level of market uncertainty and the degree of algorithmic versus human trading, they show that liquidity increases initially as AT rises to about $10 \%$ share of the market. The authors also demonstrate that statistical arbitrage can lead to significant deviations in asset prices from fundamentals.

Stübinger and Schneider (2019) uses a mean-reverting jump-diffusion model applied to high-frequency data of the S\&P 500 constituents to identify temporary market anomalies during the first minutes of a trading day. Using data from January 1998-December 2015, the authors show that a statistical arbitrage strategy to select stocks based on overnight price gaps delivers statistically and economically significant returns of $51 \%$ and an annualized Sharpe ratio of 2.38 after transaction costs which is shown to be superior to several alternative existing quantitative strategies.

Finally, using high-frequency data from several sources Petrov et al. (2019) propose a novel intraday instantaneous volatility measure which utilises sequences of drawdowns and drawups as indicators of high-frequency activity of financial markets. The authors use their measure to uncover weekly seasonal patterns in volatility for three Forex and one Bitcoin exchange rates, as well as a stock market index. The provided volatility estimation method can be used for risk-management independent of the discreteness and the type of analysed high-frequency data.

This volume includes a wide variety of theoretical and empirical contributions that address a wide range of issues and topics related to computational finance. The published papers consider asset pricing in general with applications to bond pricing, see Dunne (2019), commodity modelling, see Cheng et al. (2019), and derivatives pricing, see Létourneau and Stentoft (2019), Reesor and Marshall (2020) and Stentoft (2019); uses calibration techniques, see Van Dijk et al. (2018); and considers issues related to hedging, see Dunne (2019). The published papers develop new multivariate models, see Cheng et al. (2019), considers option pricing in this challenging setting, see Reesor and Marshall (2020), and addresses issues related to risk management, see Cheng et al. (2019), Forsyth and Vetzal (2019), and Van Dijk et al. (2018). The volume contains several papers that use simulation, see Dunne (2019), Létourneau and Stentoft (2019), Mukerji et al. (2019), and Stentoft (2019), and papers that provide new ways to model volatility, see Cheng et al. (2019), and for estimating this, see Petrov et al. (2019).

Funding: This research received no external funding

Acknowledgments: I wish to thank all the contributors and the editors at the Journal of Risk and Financial Management for supporting this special issue.

Conflicts of Interest: The author declares no conflict of interest. 


\section{References}

Cheng, Yuyang, Marcos Escobar-Anel, and Zhenxian Gong. 2019. Generalized Mean-Reverting 4/2 Factor Model. Journal of Risk and Financial Management 12: 159. [CrossRef]

Dunne, Peter G. 2019. Positive Liquidity Spillovers from Sovereign Bond-Backed Securities. Journal of Risk and Financial Management 12: 58. [CrossRef]

Forsyth, Peter A., and Kenneth R. Vetzal. 2019. Defined Contribution Pension Plans: Who Has Seen the Risk? Journal of Risk and Financial Management 12: 70. [CrossRef]

Létourneau, Pasvcal, and Lars Stentoft. 2019. Bootstrapping the Early Exercise Boundary in the Least-Squares Monte Carlo Method. Journal of Risk and Financial Management 12: 190.

Longstaff, Francis A., and Eduardo S. Schwartz. 2001. Valuing American Options by Simulation: A Simple Least-Squares Approach. Review of Financial Studies 14: 113-47. [CrossRef]

Mukerji, Purba, Christine Chung, Timothy Walsh, and Bo Xiong. 2019. The Impact of Algorithmic Trading in a Simulated Asset Market. Journal of Risk and Financial Management 12: 68. [CrossRef]

Petrov, Vladimir, Anton Golub, and Richard Olsen. 2019. Instantaneous Volatility Seasonality of High-Frequency Markets in Directional-Change Intrinsic Time. Journal of Risk and Financial Management 12: 54. [CrossRef]

Reesor, R. Marm, and T. James Marshall. 2020. Forest of Stochastic Trees: A Method for Valuing Multiple Exercise Options. Journal of Risk and Financial Management 13: 95. [CrossRef]

Stentoft, Lars. 2019. Efficient Numerical Pricing of American Call Options Using Symmetry Arguments. Journal of Risk and Financial Management 12: 59. [CrossRef]

Stübinger, Johannes, and Lucas Schneider. 2019. Statistical Arbitrage with Mean-Reverting Overnight Price Gaps on High-Frequency Data of the S\&P 500. Journal of Risk and Financial Management 12: 51.

Van Dijk, Marcel T. P., Cornelis S. L. De Graaf, and Cornelis W. Oosterlee. 2018. Between $P$ and $Q$ : The $P Q$ Measure for Pricing in Asset Liability Management. Journal of Risk and Financial Management 11: 67. [CrossRef]

(C) 2020 by the author. Licensee MDPI, Basel, Switzerland. This article is an open access article distributed under the terms and conditions of the Creative Commons Attribution (CC BY) license (http:/ / creativecommons.org/licenses/by/4.0/). 